\title{
Review
}

\section{Microglial Gene Expression Alterations in the Brains of Patients with Psychiatric Disorders}

\author{
Mai Sakai ${ }^{\mathrm{a}, \mathrm{b}}$, Yuta Takahashi ${ }^{\mathrm{a}, \mathrm{b}, \mathrm{c}}$, Zhiqian $\mathrm{Yu}^{\mathrm{a}, \mathrm{b}, \mathrm{d}}$ and Hiroaki Tomita ${ }^{\mathrm{a}, \mathrm{b}, \mathrm{d}, \mathrm{e}, *}$ \\ ${ }^{a}$ Department of Disaster Psychiatry, Graduate School of Medicine, Tohoku University, Sendai, Japan \\ ${ }^{\mathrm{b}}$ Department of Disaster Psychiatry, International Research Institute of Disaster Science, Tohoku University, \\ Sendai, Japan \\ ${ }^{c}$ Department of Psychiatry, Graduate School of Medicine, Tohoku University, Sendai, Japan \\ ${ }^{\mathrm{d}}$ Group of Mental Health Promotion, Department of Preventive Medicine and Epidemiology, \\ Tohoku Medical Megabank Organization, Tohoku University, Sendai, Japan \\ ${ }^{\mathrm{e}}$ Department of Disaster Psychiatry, Tohoku University Hospital, Tohoku University, Sendai, Japan
}

\begin{abstract}
As recent studies have shown that microglia play a key role in inflammation and immunological challenges as well as have broader roles in synaptic modulation in the brain, studies on psychiatric disorders have increasingly focused on microglia. Microglial abnormalities have consistently been observed in psychiatric postmortem brain studies, including altered microglial activation and changes in the protein and mRNA expression levels of microglial marker molecules, such as major histocompatibility complex, class II, DR (HLA-DR), complement receptor type 3 (CD11b), ionized calcium binding adaptor molecule 1 (IBA-1), macrosialin (CD68) and glucose transporter type 5 (GLUT5). Microglial abnormalities have also been observed in positron emission tomography (PET) studies. Recent advances in omics-based microglial gene expression profiling of psychiatric brains may elucidate microglial involvement in the pathogeneses of psychiatric disorders. In the present paper, we review the current status of research on expression profiling of microglia-relevant molecules in psychiatric postmortem and imaging studies and we discuss future research directions.
\end{abstract}

Keywords: Microglia, cytokine, chemokine, neurotropic factor, postmortem, positron emission tomography

\section{INTRODUCTION}

Microglia are highly dynamic immune cells in the brain located within the brain parenchyma [1]. They differentiate from hematopoietic cells and then slowly turn over and are replenished by proliferation in the adult central nervous system (CNS) [2]. In the healthy brain, resting ramified microglia have neuroprotective and neurotrophic roles by dynamically interacting with other glial cells and neurons $[3,4]$. In some pathological conditions, microglia activation

\footnotetext{
*Correspondence to: Hiroaki Tomita, MD, PhD, Department of Disaster Psychiatry, International Research Institute of Disaster Science, Tohoku University, 2-1 Seiryo-Machi, Aoba-ku, Sendai 980-8573, Japan. E-mail: htomita@med.tohoku.ac.jp.
}

may support brain inflammation, leading to neuronal cell death, which is mediated by the microglial release of pro-inflammatory cytokines and reactive oxygen and nitrogen species [11]. However, microglia that are activated in response to pathological events also have immune capacity or facilitate tissue repair [5]. Microglial activation, which is characterized by morphological changes, migration, proliferation, phagocytosis, production and the release of cytokines and chemokines, is induced by purinergic receptor agonists via increasing microglial intracellular calcium [6-10].

Recent evidence indicates that microglia regulate neuronal functions in the absence of immune challenge or inflammation. Microglia are required 
for synapses to develop during embryogenesis [11] and to regulate the number of functional synapses in the neonatal brain $[12,13]$. Microglia also regulate adult neurogenesis, refine neuronal circuitry and drive oligodendrocyte differentiation during CNS remyelination [13-15]. Considering abnormalities in synaptic regulation, neuroplastic changes and neurogenesis have been implicated in the pathology of psychotic disorders [16-18]. Microglia-neuron crosstalk [19] may be a focus of future studies on psychiatric disorders.

Microglial activation has been observed in postmortem brains from patients with psychiatric disorders, including schizophrenia [20], mood disorders [21, 22], substance abuse [23], and suicidality [24]. However, detailed mechanisms of microglia involvement in the pathogeneses of psychiatric disorders have not been elucidated. Recent advances in omics technology may provide useful tools for studying the mechanism of microglia involvement. In this review, we summarize evidence supporting microglial dysfunction in psychiatric postmortem brain samples as well as discuss the current status and perspectives in omics strategies for characterizing the molecular mechanisms of microglial dysfunction in psychiatric brains.

\section{MICROGLIAL ACTIVATION IN PSYCHIATRIC POSTMORTEM BRAIN SAMPLES (FINDINGS BASED ON CONVENTIONAL MICROGLIAL MARKER MOLECULES)}

Microglia play a major role in neuronal migration, synapse formation, and neurotransmission control as well as in innate immunity and inflammation regulation within the developing and mature nervous system. In terms of the pathophysiology of neuropsychiatric disorders, previous studies have identified human microglial cell activation based on conventional microglia-specific markers, including major histocompatibility complex, class II, DR (HLA-DR), complement receptor type3 (CD11b, also known as integrin, alpha-M; ITGAM), ionized calcium binding adaptor molecule 1 (IBA1, also known as allograft inflammatory factor 1; AIF1), macrophage antigen CD68, (CD68, also known as macrosialin), and glucose transporter type 5 (GLUT5, also known as solute carrier family 2, member 5; SLC2A5) [20, 24-26], in psychiatric illnesses, which are described below. Based on these observations, theories on the neuropsychoimmunological mechanisms of neuropsychiatric disorders have been proposed, including immunological alterations that have been demonstrated in peripheral blood and cerebrospinal fluid samples from patients with psychiatric illnesses.

\section{Schizophrenia}

HLA-DR-immunostaining of postmortem brain tissue has suggested that there is microglial activation or increased microglial cellular density in schizophrenia. For example, HLA-DR-positive (i.e., activated) microglia are found in patients with lateonset schizophrenia (age older than 40 years) [20]. HLA-DR-positive microglia are increased in the frontal cortex [27-30] and temporal cortex [5, 6] of patients with chronic schizophrenia, while the brain region morphologies are not drastically altered in general [28]. HLA-DR-positive microglia in the posterior hippocampus are increased in paranoid schizophrenia patients compared with patients who have residual schizophrenia or with controls [31]. A significant increase was observed in the mean protein CD11b levels in the frontal cortex of patients with schizophrenia compared with controls [27]. CD11b is one of the most important phagocyte receptors for recognizing microbial pathogens [35], and it has been found in murine $[36,37]$ and human microglia [38]; furthermore, it is widely used as a marker for microglial identification. A qualitative assessment of IBA-1-stained microglial morphology demonstrated numerous activated microglial cells in the dorsolateral prefrontal cortex of schizophrenia patients [32]. IBA-1 is a calcium binding protein that is exclusively expressed in the microglia of murine and human brains $[48,49]$.

By contrast, several studies have provided evidence against the aforementioned proposed ideas. There were no statistically significant changes in the numbers of HLA-DR-positive cells in the anterior cingulate cortex [30, 33-35], dorsolateral prefrontal cortex [28, 33, 34], hippocampus [31, 33, 34, 36], mediodorsal thalamus [33, 34] and dentate gyrus [34] from patients with schizophrenia. CD68-positive microglia were not altered in the mediodorsal thalamus [37] and dorsolateral prefrontal cortex [38] in patients with schizophrenia. CD68 is a member of a growing family of hematopoietic mucin-like molecules that are present in macrophages/microglia, and CD68 is a marker of cell proliferation [44]. Additionally, electron microscopy and morphometry analysis showed microglial cells are activated in both the PFC and visual cortex of schizophrenia patients [39]. 
Table 1

Microglia-relevant molecule expression changes in the brains of patients with psychiatric disorders

\begin{tabular}{|c|c|c|c|c|c|c|c|}
\hline Gene Symbol & Gene Name & Disease & Brain region & mRNA & Protein & $\begin{array}{l}\text { Marker-positive } \\
\text { Cell number }\end{array}$ & Refferences \\
\hline \multirow[t]{2}{*}{$\overline{\text { IBA1 (AIF1) }}$} & $\begin{array}{l}\text { Ionized calcium binding } \\
\text { adapter molecule } 1\end{array}$ & Alcoholic & VTA, CC & - & $\bar{\uparrow}$ & - & [23] \\
\hline & $\begin{array}{l}\text { (Allograft inflammatory } \\
\text { factor 1) }\end{array}$ & Suicide & DLPFCl WM & - & - & $\uparrow$ & {$[24]$} \\
\hline \multirow[t]{2}{*}{ CD11B (ITGAM) } & \multirow{2}{*}{$\begin{array}{l}\text { Cluster of differentiation } \\
\text { molecule } 11 \text { b (Integrin, } \\
\text { alpha-M) }\end{array}$} & $\mathrm{BD}$ & PFC & $\uparrow$ & $\uparrow$ & - & {$[40]$} \\
\hline & & $\mathrm{SCZ}$ & PFC (BA 10) & $\uparrow$ & $\uparrow$ & - & [27] \\
\hline $\begin{array}{l}\text { GLUTS } \\
\quad \text { (SLC2A5) }\end{array}$ & $\begin{array}{l}\text { Glucose transporter-5 (Solute } \\
\text { carrier family } 2, \text { member } 5 \text { ) }\end{array}$ & Alcoholic & CC, Midbrain & - & $\uparrow$ & - & [23] \\
\hline \multirow[t]{3}{*}{ HLA-DR } & \multirow{3}{*}{$\begin{array}{l}\text { Class II major } \\
\text { histocompatibility complex } \\
\text { DR }\end{array}$} & $\mathrm{SCZ}$ & $\begin{array}{r}\text { DLPFC (BA 9), } \\
\text { STG (BA 22) }\end{array}$ & - & - & $\uparrow$ & [28] \\
\hline & & $\mathrm{BD}$ & PFC & - & - & $\uparrow$ & {$[40]$} \\
\hline & & $\mathrm{SCZ}$ (P vs R) & $\mathrm{Hi}$ & - & - & $\uparrow$ & {$[31]$} \\
\hline \multirow[t]{4}{*}{ IL-13 } & \multirow[t]{4}{*}{ Interleukin-1, beta } & $\mathrm{BD}$ & PFC & $\uparrow$ & $\uparrow$ & - & [40] \\
\hline & & Sucide & PFC (BA 10) & $\uparrow$ & $\uparrow$ & - & [73] \\
\hline & & Sucide & PFC (BA 8) & - & $\uparrow$ & - & \\
\hline & & SCZ & PFC (BA 10) & $\uparrow$ & $\uparrow$ & - & [27] \\
\hline IL-1RN & $\begin{array}{l}\text { Interleukin } 1 \text { receptor } \\
\text { antagonist }\end{array}$ & $\mathrm{SCZ}$ & $\mathrm{PFC}$ & - & $\downarrow$ & - & [66] \\
\hline IL-1R & interleukin-1 receptor & $\mathrm{BD}$ & PFC & $\uparrow$ & $\uparrow$ & - & [40] \\
\hline IL-4 & Interleukin 4 & Sucide & OF (BA 11) & $\uparrow$ & - & - & [74] \\
\hline \multirow[t]{2}{*}{ IL-6 (IFNB2) } & \multirow[t]{2}{*}{$\begin{array}{l}\text { Interleukin } 6 \text { (Interferon } \\
\text { beta } 2 \text { ) }\end{array}$} & $\mathrm{SCZ}$ & DLPFC(BA 46) & $\uparrow$ & - & - & [29] \\
\hline & & Sucide & PFC (BA 10) & $\uparrow$ & - & - & [73] \\
\hline IL-8 & Interleukin 8 & $\mathrm{SCZ}$ & DLPFC (BA 46) & $\uparrow$ & - & - & [29] \\
\hline \multirow[t]{3}{*}{ TNFA (TNF) } & \multirow{3}{*}{$\begin{array}{l}\text { Tumor necrosis factor, alpha } \\
\text { (Tumor necrosis factor) }\end{array}$} & Sucide & PFC (BA 10) & $\uparrow$ & $\uparrow$ & - & [73] \\
\hline & & Sucide & $\mathrm{PFC}(\mathrm{BA} 8)$ & - & $\uparrow$ & - & \\
\hline & & $\mathrm{SCZ}$ & PFC (BA 10) & $\uparrow$ & $\uparrow$ & - & [27] \\
\hline \multirow[t]{2}{*}{ tmTNF (TNF) } & \multirow{2}{*}{$\begin{array}{l}\text { Transmembrane tumor } \\
\text { necrosis factor-alpha } \\
\text { (Tumor necrosis factor) }\end{array}$} & MDD & DLPFC (BA 46) & - & $\uparrow$ & - & [70] \\
\hline & & BD & $\mathrm{AC}(\mathrm{BA} 24)$ & - & $\uparrow$ & - & {$[67]$} \\
\hline $\begin{array}{l}\text { TNFRSF1A } \\
\text { (TNFR1) }\end{array}$ & $\begin{array}{l}\text { Tumor necrosis factor } \\
\text { receptor superfamily, } \\
\text { member 1A (Tumor } \\
\text { necrosis factor receptor 1) }\end{array}$ & $\mathrm{SCZ}$ & $\begin{array}{l}\text { AC (BA 24), } \\
\text { DLPFC (BA 46) }\end{array}$ & $\uparrow$ & - & - & \\
\hline $\begin{array}{l}\text { TNFRSF1B } \\
\text { (TNFR2) }\end{array}$ & $\begin{array}{l}\text { Tumor necrosis factor } \\
\text { receptor superfamily, } \\
\text { member 1B (Tumor } \\
\text { necrosis factor receptor 2) }\end{array}$ & $\mathrm{MDD}, \mathrm{BD}$ & DLPFC(BA 46) & $\downarrow$ & - & - & \\
\hline \multirow[t]{4}{*}{ QUIN } & \multirow[t]{4}{*}{ Quinolinic acid } & $\mathrm{SCZ}$ & Hi (CA1) & - & - & $\downarrow$ & [36] \\
\hline & & MDD & $\begin{array}{l}\mathrm{Hi} \text { (right CA1 and } \\
\text { left CA2/3) }\end{array}$ & - & $\downarrow$ & - & [69] \\
\hline & & UD & $\begin{array}{l}\mathrm{Hi} \text { (right CA1 } \\
\text { subregion) }\end{array}$ & - & $\downarrow$ & - & \\
\hline & & $\mathrm{BD}$ & $\begin{array}{l}\text { Hi (right CA1 } \\
\text { subregion) }\end{array}$ & - & $\downarrow$ & - & \\
\hline \multirow[t]{2}{*}{ NFKB1 } & \multirow{2}{*}{$\begin{array}{l}\text { Nuclear factor kappa-B, } \\
\text { subunit (Nuclear factor of } \\
\text { kappa light polypeptide } \\
\text { gene enhancer in B-cells 1) }\end{array}$} & $\mathrm{BD}$ & PFC (BA 10) & $\uparrow$ & $\uparrow$ & - & [40] \\
\hline & & $\mathrm{SCZ}$ & PFC (BA 10) & $\uparrow$ & $\uparrow$ & - & [27] \\
\hline RELA (NFKB3) & $\begin{array}{l}\text { V-Rel Avian } \\
\text { Reticuloendotheliosis Viral } \\
\text { Oncogene Homolog A } \\
\text { (Nuclear factor kappa-B, } \\
\text { subunit 3) }\end{array}$ & $\mathrm{BD}$ & PFC (BA 10) & $\uparrow$ & $\uparrow$ & - & [40] \\
\hline S100A8 (CFAG) & $\begin{array}{l}\text { S100 Calcium-Binding } \\
\text { Protein A8 (Cystic fibrosis } \\
\text { antigen) }\end{array}$ & $\mathrm{SCZ}$ & $\begin{array}{l}\text { DLPFC GM (BA } \\
\text { 9) }\end{array}$ & - & $\uparrow$ & - & {$[42]$} \\
\hline
\end{tabular}


Table 1

(Continued)

\begin{tabular}{|c|c|c|c|c|c|c|c|}
\hline Gene Symbol & Gene Name & Disease & Brain region & mRNA & Protein & $\begin{array}{c}\text { Marker-positive } \\
\text { Cell number }\end{array}$ & $\overline{\text { Refferences }}$ \\
\hline S100A9 (CAGB) & $\begin{array}{l}\text { S100 Calcium-Binding } \\
\text { Protein A9 (Calgranulin B) }\end{array}$ & $\mathrm{SCZ}$ & $\begin{array}{l}\text { DLPFC GM } \\
\text { (BA 9) }\end{array}$ & - & $\uparrow$ & - & \\
\hline NR1 (GRIN1) & $\begin{array}{l}\text { N-Methyl-D-Aspartate } \\
\text { Receptor channel, subunit } \\
\text { zeta-1 (Glutamate receptor, } \\
\text { ionotropic, } \\
\text { N-methyl-D-Aspartate, } \\
\text { subunit 1) }\end{array}$ & $\mathrm{BD}$ & PFC & $\downarrow$ & $\downarrow$ & - & {$[40]$} \\
\hline NR3A (GRIN3A) & $\begin{array}{l}\text { N-methyl D-aspartate } \\
\text { receptor subtype 3A } \\
\text { (Glutamate receptor, } \\
\text { ionotropic, } \\
\text { N-methyl-D-Aspartate 3A) }\end{array}$ & BD & PFC & $\downarrow$ & $\downarrow$ & - & \\
\hline MYD88 & $\begin{array}{l}\text { Myeloid differentiation } \\
\text { primary response gene } 88\end{array}$ & $\mathrm{BD}$ & PFC & $\uparrow$ & $\uparrow$ & - & \\
\hline INOS (NOS2A) & $\begin{array}{l}\text { Nitric oxcide synthase, } \\
\text { inducible (Nitric oxide } \\
\text { synthase } 2 \mathrm{~A} \text { ) }\end{array}$ & $\mathrm{BD}$ & $\mathrm{PFC}$ & $\uparrow$ & $\uparrow$ & - & \\
\hline \multirow[t]{3}{*}{ BDNF } & $\begin{array}{l}\text { Brain-derived neurotrophic } \\
\text { factor }\end{array}$ & $\mathrm{SCZ}$ & PFC (BA 10) & $\downarrow$ & $\downarrow$ & - & [27] \\
\hline & & Sucide & $\mathrm{PFC}, \mathrm{Hi}$ & $\downarrow$ & $\downarrow$ & - & {$[75]$} \\
\hline & & Suicide (Teenage) & PFC, Hi & $\downarrow$ & $\downarrow(\mathrm{PFC})$ & - & [76] \\
\hline CORO1A & Coronin 1A & MDD & aPFC (BA 10) & - & $\downarrow$ & - & [72] \\
\hline
\end{tabular}

The table summarizes changes in the transcript and protein expression levels of microglia-relevant molecules as well as in the numbers of microglia labeled with antibodies specific to molecules in the postmortem brains of patients with psychiatric disorders. The columns represent the gene symbol (alternative gene symbol in each parenthesis), gene name (alternative gene name in each parenthesis), psychiatric disorder diagnosis, brain region, direction of change in the transcript and protein expression levels, number of microglia compared with controls, and reference. BD: bipolar disorder; MDD: major depressive disorder; UD: unipolar disorder; SCZ: schizophrenia; P vs R: paranoid type vs residual type of schizophrenia; AC: anterior cingulate cortex; aPFC: anterior prefrontal cortex; BA: Brodmann area; CC: cingulate cortex; DG: dentate gyrus; DLPFC: dorsolateral prefrontal cortex; GM: gray matter; Hi: hippocampus; OF: orbitofrontal area; PFC: prefrontal frontal cortex; SG: supragranular regions; STG: superior temporal gyrus; VTA: ventral tegmental area; WM: white matter.

\section{Bipolar disorder}

Recent studies have shown that bipolar disorder involves microglial activation. Significant increases in the protein and mRNA levels of CD11b have been observed along with increased HLA-DR-positive microglia in the postmortem frontal cortices of bipolar disorder patients [40].

However, the density of IBA-1-stained microglia did not differ among bipolar disorder and control patients, which appears to contrast with the above study [32]. Another study also reported no significant differences in the HLA-DR-positive microglial density for bipolar I disorder patients compared with controls [33].

\section{Depression}

Because evidence supports microglial involvement in the pathogeneses of depression, Yirmiya, RN et al proposed that depression is a microglial disease [21]. A postmortem study demonstrated strong activation of HLA-DR-positive microglia in an old, affective disorder patient by immunohistochemistry [20]. Significant HLA-DR-positive microgliosis has been observed in the dorsolateral prefrontal cortex, anterior cingulate cortex and mediodorsal thalamus of suicidal patients with major depression, and a similar trend has been observed in the hippocampus [33]. Microgliosis is an accumulation of microglial cells as a reaction to parenchymal injury, which is accompanied with cellular morphological changes, such as the enlargement of cellular processes [41].

However, three other studies reported no differences between depressed patients and controls in IBA-1- and CD68- [24], HLA-DR- [33], and CD68[42] positive microglial density.

\section{Alcoholic patients}

The immune system in the form of systemic and CNS inflammation has emerged in the field of alcohol abuse and alcoholism [43]; however, microglial 
involvement in alcoholic patients has not been well characterized. Thus far, only one research study showed that both IBA-1 and GluT5 were increased in the cingulate cortex of alcoholic brains relative to controls [23].

\section{Suicidality}

Recent postmortem studies reported that microgliosis, labeled by HLA-DR, was increased in two schizophrenic patients in the anterior cingulate cortex and mediodorsal nucleus of the thalamus; both of these patients, who had acute psychosis, committed suicide [34]. Additionally, microgliosis was observed in the dorsolateral prefrontal cortex and mediodorsal nucleus for suicide cases [33]. Additionally, one study indicated that the gene expression of IBA-1 and mRNA expression of CD45 were significantly increased in the dorsal anterior cingulate cortex for depressed patients who committed suicide [44]. However, the study reported no differences in the densities of resting or activated microglia in the dorsal or ventral prefrontal white matter, but the density of perivascular cells in dorsal white matter was higher between suicide and non-suicide cases, whereas the densities of activated microglial cells were higher ventrally than dorsally among suicide patients as well higher dorsally than ventrally among non-suicide cases [24]. Other studies also indicated that there are no significant microglial densities in the dorsolateral prefrontal cortex, anterior cingulate cortex, mediodorsal thalamus, and hippocampus of schizophrenic and depressive patients who committed suicide compared with non-suicidal cases [33]

\section{Autism}

Postmortem studies showed that the IBA-1positive microglial somal volume in white matter and microglial cell density in gray matter were increased in a case with autism compared with control cases [45]. In a postmortem study of adult autistic patients, 2 of 8 autism brains displayed significant IBA-1stained microglial activation in the amygdala [46]. HLA-DR-positive microglia were observed in the postmortem cerebellum of an autism patient [47]. The total densities of IBA-1-positive microglia were significantly greater in the cerebral cortex of autistic individuals, ranging from 3 to 22 , compared with the control [48]. Additionally, the autism brains exhibited increased short-distance, microglia-neuron interactions (microglia-neuron spatial clustering) in the dorsolateral prefrontal cortex [49].

\section{MICROGLIAL ACTIVATION IN PSYCHIATRIC DISORDERS BASED ON IMAGING STUDIES}

Microglial activation in the brain of psychiatric patients, especially patients with schizophrenia, has been evaluated in several positron emission tomography (PET)-based neuroimaging studies utilizing microglia-specific radiotracers, such as (R)$\left[{ }^{11} \mathrm{C}\right] \mathrm{PK} 11195$. (R)-[ $\left.{ }^{11} \mathrm{C}\right] \mathrm{PK} 11195$ binds selectively to the peripheral benzodiazepine receptor, which is also known as the mitochondrial $18 \mathrm{kDa}$ translocator protein (TSPO). (R)-[ $\left[{ }^{11} \mathrm{C}\right] \mathrm{PK} 11195$ binding was significantly higher in the hippocampus of schizophrenic patients (five patients were paranoid schizophrenia and 2 patients had brief psychotic disorder not otherwise specified) [50]. (R)- $\left[{ }^{11} \mathrm{C}\right] \mathrm{PK} 11195$ was also significantly higher in the total gray matter of schizophrenic patients during acute psychotic episodes that were early in the disease course [51]. Another PET study on a novel positron emission tomography (PET) ligand, $\left[{ }^{11} \mathrm{C}\right] \mathrm{DAA} 1106$, instead indicated that there were no significant microglial alterations in any of the brain regions between patients with chronic schizophrenia and control subjects. There was no significant difference between $\left[{ }^{11}\right.$ C]DAA1106 binding of the cortical regions of normal controls and patients with schizophrenia, whereas the patients had a positive correlation between cortical [11C]DAA1106 binding and positive symptom scores [52].

PET-based evaluation of microglial abnormalities has rarely been used for other psychiatric disorders. Thus far, a study on bipolar disorder reported a significantly increased $\left[{ }^{11} \mathrm{C}\right]-(\mathrm{R})-\mathrm{PK} 11195$ binding potential in the right hippocampus of disease patients compared with controls. Although the same trend was observed in the left hippocampus, this difference was not statistically significant [53]. To the best of our knowledge, there have been no reports on PET-based evaluation of microglial abnormalities in patients with alcoholism. PET imaging revealed microglial activation in autistic subjects compared to control subjects. Significantly higher (R)-[ $\left[{ }^{11} \mathrm{C}\right] \mathrm{PK} 11195$ levels were observed in the cerebellum, midbrain, pons, fusiform gyri, and anterior cingulate and orbitofrontal cortices from young adult subjects with autistic spectrum disorder compared with control subjects [54]. 


\section{MICROGLIAL GENE EXPRESSION ABNORMALITIES IN PSYCHIATRIC POSTMORTEM BRAIN SAMPLES AND POTENTIAL MICROGLIAL INVOLVEMENT}

In addition to the aforementioned conventional microglial marker molecules, microglia exert their functions via transcriptional regulations of various genes. For example, microglia play major roles in neuroimmunological and neuroinflammatory processes via transcriptional regulation of proor anti-inflammatory cytokines and neurotrophic factors, which also play important roles in the development of psychiatric disorders [55]. Microglia can differentiate into M1 and M2 phenotypes during these processes [56]. The M1 phenotype is associated with enhanced antigen presentation and secretion of proinflammatory cytokines, such as interleukin-1 (IL-1), IL-6, IL-8 and tumor necrosis factor- $\alpha$ (TNF $\alpha)$. The M1 phenotype is also associated with nuclear factorkappa B (NF-кB), a transcription factor that plays a major role in regulating pro-inflammatory genes as well as in upregulating myeloid differentiation primary response gene 88 (MyD88), which facilitates NF-кB activities in combination with toll-like receptors [57, 58]. The M1 phenotype is also characterized by activated nitric oxide synthase (iNOS) expression $[5,15,59,60]$. In contrast, the M2 phenotype secretes anti-inflammatory cytokines IL-4, IL-5 and IL-10 as well as bone morphogenetic protein 7 (BMP-7), which suppresses the expression levels of pro-inflammatory cytokines. The M2 phenotype is also characterized by the expression of trophic factors, including brain-derived neurotrophic factor (BDNF), vascular endothelial growth factor (VEGF), glial cell-derived neurotrophic factor (GDNF), insulin-like growth factor gene 1 (IGF-1), transforming growth factor $\beta$ (TGF- $\beta$ ), and hepatocyte growth factor (HGF) [5].

In addition to the molecules related to $\mathrm{M} 1$ and $\mathrm{M} 2$ microglia, S100A8- and S100A9-positive microglia are observed in brain samples following cerebral infarction and other neurological disorders [72, 73]. S100A8 and S100A9 belong to the macrophagerelated protein-100 family of calcium binding proteins, which inhibit pro-inflammatory functions [61-63]. QUIN, an endogenous modulator with agonistic properties against N-methyl-D-aspartate, which is produced by microglia, plays a role in the pathogenesis of several major neurological diseases $[64,65]$. Evidence supports that microglial abnor- malities are accompanied by inflammatory cytokines, which may be responsible for the abnormalities in neural development or psychiatric disorder functionality. In the brain, only microglia, not neurons or astrocytes, produce detectable QUIN levels [65].

\section{Schizophrenia}

In frontal cortex samples from SZ patients, the IL-1 $\beta$ protein level was significantly increased [27], whereas IL-1 receptor antagonist (IL-1RA) protein, which is mostly enriched in the prefrontal cortex, was significantly reduced in the schizophrenic brain samples [66]. The IL-6 and IL- 8 mRNA expression levels were also significantly upregulated in the dorsolateral prefrontal cortices from schizophrenic patients [29]. The TNF $\alpha$ mRNA and protein levels were also significantly increased in frontal cortex samples from schizophrenic patients [27]. Additionally, the TNF receptor type I (TNFR1) mRNA level was significantly increased in both the anterior cingulate and dorsolateral prefrontal cortices of patients with schizophrenia [67]. The protein expression levels of M1-related nuclear transcription factor subunits, NF-кBp50 and NF-кBp65, were significantly increased in the frontal cortices of schizophrenia patients. In contrast, the protein and mRNA levels of the M2-related growth factor BDNF were significantly reduced in the frontal cortices of schizophrenia patients [27]. The protein expression levels of other microglial marker chemokines, S100A8/A9, were significantly higher in the dorsolateral prefrontal cortices of patients with schizophrenia [42]. Additionally, there were significantly more QUIN-immunoreactive microglial cells in the CA1 hippocampal subregions of schizophrenia patients. However, no significant changes in quinolinic acid (QUIN)-immunoreactive microglial cells were observed in the hippocampus CA2/3 and dentate gyrus regions of patients with schizophrenia [36].

\section{Bipolar disorder}

A postmortem study demonstrated significantly elevated mRNA and protein levels of IL-1 $\beta$, IL-1R and MyD88 in the frontal cortices of bipolar disorders patients [40]. The tmTNF $\alpha$ levels were increased in the postmortem anterior cingulate of bipolar disorder patients [67]. The TNFR2 mRNA level was significantly decreased in the dorsolateral prefrontal cortices of bipolar and major depressive disorder patients [67]. The nuclear protein and mRNA levels 
of microglial NFKB1 (p50) and NF-кBp65 [40] were significantly increased in the postmortem frontal cortices of bipolar disorder patients. Recombinant $\mathrm{N}$-methyl-d-aspartate receptor subtypes NR-1A and NR-3A were expressed in the microglia [68] and the corresponding mRNA and protein levels were significantly decreased in the frontal cortices of bipolar disorder patients compared to controls [40]. M1 Microglia phenotype marker iNOS mRNA and protein levels were significantly increased in the frontal cortices of bipolar disorder patients compared with controls [40]. Additionally, QUIN staining was reduced in both unipolar and bipolar disorders in the right CA1 field as well as in depressed patients in the right CA1 and left CA2/3 of the hippocampus [69].

\section{Depression}

Several postmortem studies reported that the transmembrane TNF (tmTNF) protein level was significantly increased in the dorsolateral prefrontal cortex but not increased in the anterior cingulate cortex of major depression disorder patients. There was no change in the soluble TNF (sTNF) levels in either brain region [70]. Patients with bipolar disorder and major depressive disorder had significant reductions in the TNFR2 mRNA expression in the dorsolateral prefrontal cortex without a reduction in the anterior cingulate cortex levels [67]. Additionally, microgliaspecific actin-binding protein coronin-1a (CORO1A) [71] was decreased in the anterior prefrontal cortices of patients with major depressive disorder [72].

\section{Suicidality}

Of the pro-inflammatory cytokines, the IL- $1 \beta$, IL-6 and TNF $\alpha$ mRNA and protein levels were significantly higher in the prefrontal cortices of suicide victims compared with healthy control brains [73]. Analysis of covariance revealed no significant difference in the IL-1 $\beta$, IL- 6 and TNF $\alpha$ expression levels in the orbitofrontal cortex between controls and suicidal patients, whereas there were significant differences in the orbitofrontal area in the expression levels of an anti-inflammatory cytokine, IL-4, between suicide victims and controls. Higher IL-4 expression levels were observed in women [74]. The BDNF protein and mRNA levels were significantly reduced in both the prefrontal cortex and hippocampus of suicide subjects [75]. The BDNF protein expression level was significantly decreased in the prefrontal cortex of teenage suicide victims, whereas no change was observed in the hippocampus [76]. Additionally, the mRNA expression of both BDNF and its receptor, tropomyosin receptor kinase $B(\operatorname{TrkB})$, were significantly decreased in the prefrontal cortex and hippocampus of teenage suicide victims [76].

\section{COMPREHENSIVE GENE EXPRESSION PROFILES OF MICROGLIA AND GENE FUNCTION}

Thus far, a number of gene expression profiles of postmortem brain tissues from patients with psychiatric disorders have been reported for schizophrenia, bipolar disorder, depression, and autism. Among them, the cell type specificities for neurons [77-81] and oligodendrocytes [82, 83] have thoroughly been discussed. However, microglia-related gene expression profiles have received little attention. In addition to the aforementioned conventional microglial markers, cytokines and growth factors, various other microglia-specific molecules may be involved in neuroinflammatory aspects of the pathogeneses of psychiatric disorders. Evaluation of the comprehensive gene expression profiles of microglia may help clarify the role of microglia in psychiatric disorders.

To better understand how microglial gene expression profiles differ between schizophrenic patients and controls, we selected tentative microglial gene expression profiles based on the gene expression profile of isolated microglia from mice and then compared them with gene expression profiles that are specific to the major neuropsychiatric diseases. Using an Illumina BeadChip-based gene expression profile of microglia from C57BL/6 murine brains [84], 6,319 transcripts with signal intensities greater than 500 were considered to have high levels of detectable transcripts in microglia. However, based on Illumina BeadChip-based C57BL/6 prefrontal cortex microarray data [85] from the PubMed GEO DataSet, 22,024 transcripts had signal intensities of less than 500, which is considered low in the prefrontal cortex. The overlapped 2,024 transcripts (1,814 genes) were defined as microglia-dominant transcripts (genes), which are highly expressed in microglia and relatively less expressed in prefrontal cortex samples.

The list of microglia-dominant transcripts was compared with the gene expression profiles of postmortem prefrontal cortex samples from schizophrenia, bipolar disorder, and major depression patients provided by Stanley Foundation Brain Collection to estimate how microglia-related transcripts are 
related to the pathogeneses of psychiatric disorders. The gene expression profiles of each psychiatric disorder were compared with controls, and altered genes were considered increased or suppressed with a fold change of $>1.2$ or $<0.833$, respectively. Among whole detectable genes (8,583), 1,319, 1,636 and 1,312 genes were increased in schizophrenia, bipolar disorder and depression, respectively, while $1,263,1,253$ and 1,206 genes were suppressed in each psychiatric disorder, respectively. Of these, 391 and 278 genes were commonly increased and suppressed, respectively, in all three diseases. Among the above increased genes in schizophrenia, bipolar disorder and depression, 134 (10.2\%), 177 (10.9\%) and $158(12.0 \%)$ genes overlapped with the 1,814 microglia-dominant genes. Likewise, among the aforementioned increased genes in schizophrenia, bipolar disorder and depression, 138 (10.9\%), 113 (9.0\%) and $102(8.5 \%)$ genes overlapped with the microglia-dominant genes. Among 391 commonly increased genes and 278 commonly suppressed genes in all three diseases, $39(10.0 \%)$ and $23(8.3 \%)$ genes overlapped with the microglia-dominant genes. The proportions of altered microglia-specific genes in each psychiatric disorder were $8-12 \%$, which is reasonably comparable to the microglia $(10-15 \%)$ composition of all brain cells [86]. These preliminary data suggest that evaluating postmortem brain omics data, including gene expression profiles, while focusing on microglia may provide useful insights that have otherwise been masked by the cellular heterogeneity of the tissue characteristics.

\section{PERSPECTIVES}

As summarized in previous descriptions, multiple studies based on the microglial marker-based evaluation of postmortem brains have indicated that microglia are generally activated in the brains of patients with schizophrenia, bipolar disorder, and major depression, especially in suicidal cases. These findings were supported by multiple PET studies. Further, multiple studies have indicated that M1type marker expression levels in microglia were increased whereas M2-type markers were decreased in general in the postmortem brains of patients with psychiatric disorders. Although some studies do not support microglial activation in psychiatric postmortem brains, few studies demonstrate the opposite direction of change. Considering the heterogeneity even in a single psychiatric disorder, such as schizophrenia, bipolar disorder, and major depressive disorder, the previous findings may indicate that microglia are activated in certain types and processes of psychiatric disorders.

In addition to the heterogeneity of the disorder, several features need to be considered in postmortem brain studies of psychiatric disorders. For example, a previous study indicated that the postmortem interval was significantly correlated with the ramified cell numbers in the anterior cingulate cortex and dorsolateral prefrontal cortices as well as with the ameboid cell density in the hippocampus [34]. Notably, the effect of postmortem interval on the gene expression profile in a postmortem brain is generally low if the interval is shorter than 48 hours. However, the agonal condition and brain tissue $\mathrm{pH}$ more drastically impact the gene expression profile [87]. Evaluations of the postmortem interval, agonal factor and tissue $\mathrm{pH}$ need to be considered when assessing the molecular expression profiles of microglia in postmortem brain tissue samples from psychiatric patients.

Autoimmune processes and inflammatory component alterations have been implicated in the pathogenesis of psychiatric disorders based on postmortem brain analyses. Detailed molecular mechanisms of microglial involvement in the pathogeneses of psychiatric disorders have yet to be elucidated. On the other hand, substantial gene expression profiling of psychiatric postmortem brains has been conducted, and some expression profiling studies have focused on the cellular specificities of neurons and oligodendrocytes. However, microgliaspecific gene expression profiles have received little attention. Application of omics technologies to microglial gene expression profiling of psychiatric brains may elucidate distinct mechanisms of microglial involvement in the pathogeneses of psychiatric disorders.

\section{ACKNOWLEDGMENTS}

This work was partially supported by a grant-inaid for scientific research on innovative areas (No. 24116007) from the Ministry of Education, Culture, Sports, Science, and Technology of Japan, Health and Labour Sciences Research Grants research on psychiatric and neurological diseases and mental health (H19-kokoro-ippan-001), and an Intramural Research Grant (No. 21-9) for Neurological and Psychiatric Disorders from the National Center of Neurology and Psychiatry. All authors declare no 
biomedical financial interests or potential conflicts of interest.

\section{REFERENCES}

[1] Perry VH, Crocker PR, Gordon S. The blood-brain barrier regulates the expression of a macrophage sialic acid-binding receptor on microglia. J Cell Sci. 1992:101(Pt 1):201-207.

[2] Eglitis MA, Mezey E. Hematopoietic cells differentiate into both microglia and macroglia in the brains of adult mice. Proc Natl Acad Sci USA. 1997:94(8):4080-4085.

[3] Polazzi E, Contestabile A. Reciprocal interactions between microglia and neurons: From survival to neuropathology. Rev Neurosci. 2002:13(3):221-242.

[4] Streit WJ. Microglia as neuroprotective, immunocompetent cells of the CNS. Glia. 2002:40(2):133-139.

[5] Fumagalli M, Lecca D, Abbracchio MP. Role of purinergic signalling in neuro-immune cells and adult neural progenitors. Front Biosci (Landmark Ed). 2011:16:2326-2341.

[6] Light AR, et al. Purinergic receptors activating rapid intracellular $\mathrm{Ca}$ increases in microglia. Neuron Glia Biol. 2006:2(2):125-138.

[7] Davalos D, et al. ATP mediates rapid microglial response to local brain injury in vivo. Nat Neurosci. 2005:8(6):752-758.

[8] Toescu EC, et al. Long-term activation of capacitative $\mathrm{Ca} 2+$ entry in mouse microglial cells. Neuroscience. 1998:86(3):925-935.

[9] Hanisch UK, Kettenmann H. Microglia: Active sensor and versatile effector cells in the normal and pathologic brain. Nat Neurosci. 2007:10(11):1387-1394.

[10] Michelucci A, et al. Characterization of the microglial phenotype under specific pro-inflammatory and antiinflammatory conditions: Effects of oligomeric and fibrillar amyloid-beta. J Neuroimmunol. 2009:210(1-2):3-12.

[11] Paolicelli RC, et al. Synaptic pruning by microglia is necessary for normal brain development. Science. 2011:333(6048):1456-1458.

[12] Ji K, et al. Microglia actively regulate the number of functional synapses. PLoS One. 2013:8(2):e56293.

[13] Schafer DP, et al. Microglia sculpt postnatal neural circuits in an activity and complement-dependent manner. Neuron. 2012:74(4):691-705.

[14] Sierra A, et al. Microglia shape adult hippocampal neurogenesis through apoptosis-coupled phagocytosis. Cell Stem Cell. 2010:7(4):483-495.

[15] Miron VE, et al. M2 microglia and macrophages drive oligodendrocyte differentiation during CNS remyelination. Nat Neurosci. 2013:16(9):1211-128.

[16] Duman RS, Aghajanian GK. Synaptic dysfunction in depression: Potential therapeutic targets. Science. 2012:338(6103):68-72.

[17] Eyre H, Baune BT. Neuroplastic changes in depression: A role for the immune system. Psychoneuroendocrinology. 2012:37(9):1397-1416.

[18] Coyle JT, et al. Glutamatergic synaptic dysregulation in schizophrenia: Therapeutic implications. Handb Exp Pharmacol. 2012:(213):267-295.

[19] Kettenmann H, Kirchhoff F, Verkhratsky A. Microglia: New roles for the synaptic stripper. Neuron. 2013:77(1):10-18.

[20] Bayer TA, et al. Evidence for activation of microglia in patients with psychiatric illnesses. Neurosci Lett. 1999:271(2):126-128.

[21] Yirmiya R, Rimmerman N, Reshef R., Depression as a microglial disease. Trends Neurosci. 2015:38(10):637-658.
[22] Stertz L, Magalhaes PV, Kapczinski F. Is bipolar disorder an inflammatory condition? The relevance of microglial activation. Curr Opin Psychiatry. 2013:26(1):19-26.

[23] He J. Crews FT. Increased MCP-1 and microglia in various regions of the human alcoholic brain. Exp Neurol. 2008:210(2):349-358.

[24] Schnieder TP, et al. Microglia of prefrontal white matter in suicide. J Neuropathol Exp Neurol. 2014:73(9):880-890.

[25] Horikoshi Y, et al. Human GLUT5 immunolabeling is useful for evaluating microglial status in neuropathological study using paraffin sections. Acta Neuropathol. 2003:105(2):157-162.

[26] Lee YB, Nagai A, Kim SU. Cytokines, chemokines, and cytokine receptors in human microglia. J Neurosci Res. 2002:69(1):94-103

[27] Rao JS, et al. Increased neuroinflammatory and arachidonic acid cascade markers, and reduced synaptic proteins, in the postmortem frontal cortex from schizophrenia patients. Schizophr Res. 2013:147(1):24-31.

[28] Radewicz K, et al. Increase in HLA-DR immunoreactive microglia in frontal and temporal cortex of chronic schizophrenics. J Neuropathol Exp Neurol. 2000:59(2):137150.

[29] Fillman SG, et al. Increased inflammatory markers identified in the dorsolateral prefrontal cortex of individuals with schizophrenia. Mol Psychiatry. 2013:18(2):206-214.

[30] Wierzba-Bobrowicz T, et al. Quantitative analysis of activated microglia, ramified and damage of processes in the frontal and temporal lobes of chronic schizophrenics. Folia Neuropathol. 2005:43(2):81-89.

[31] Busse S, et al. Different distribution patterns of lymphocytes and microglia in the hippocampus of patients with residual versus paranoid schizophrenia: Further evidence for disease course-related immune alterations? Brain Behav Immun. 2012:26(8):1273-1279.

[32] Hercher C, Chopra V, Beasley CL. Evidence for morphological alterations in prefrontal white matter glia in schizophrenia and bipolar disorder. J Psychiatry Neurosci. 2014:39(6):376-385.

[33] Steiner J, et al. Immunological aspects in the neurobiology of suicide: Elevated microglial density in schizophrenia and depression is associated with suicide. J Psychiatr Res. 2008:42(2):151-157.

[34] Steiner J, et al. Distribution of HLA-DR-positive microglia in schizophrenia reflects impaired cerebral lateralization. Acta Neuropathol. 2006:112(3):305-316.

[35] Wierzba-Bobrowicz T, et al. Degeneration of microglial cells in frontal and temporal lobes of chronic schizophrenics. Folia Neuropathol. 2004:42(3):157-165.

[36] Gos $\mathrm{T}$, et al. Reduced microglial immunoreactivity for endogenous NMDA receptor agonist quinolinic acid in the hippocampus of schizophrenia patients. Brain Behav Immun. 2014:41:59-64.

[37] Falke E, Han LY, Arnold SE. Absence of neurodegeneration in the thalamus and caudate of elderly patients with schizophrenia. Psychiatry Res. 2000:93(2):103-110.

[38] Arnold SE, et al. Absence of neurodegeneration and neural injury in the cerebral cortex in a sample of elderly patients with schizophrenia. Arch Gen Psychiatry. 1998:55(3):225232.

[39] Uranova NA, et al. Ultrastructural damage of capillaries in the neocortex in schizophrenia. World J Biol Psychiatry. 2010:11(3):567-578.

[40] Rao JS, et al. Increased excitotoxicity and neuroinflammatory markers in postmortem frontal cortex from 
bipolar disorder patients. Mol Psychiatry. 2010:15(4): 384-392.

[41] Streit WJ, Walter SA, Pennell NA. Reactive microgliosis. Prog Neurobiol. 1999:57(6):563-581.

[42] Foster R, et al. Calprotectin in microglia from frontal cortex is up-regulated in schizophrenia: Evidence for an inflammatory process? Eur J Neurosci. 2006:24(12):3561-3356.

[43] Irwin MR, Miller AH. Depressive disorders and immunity: 20 years of progress and discovery. Brain Behav Immun. 2007:21(4):374-383.

[44] Torres-Platas SG, et al. Evidence for increased microglial priming and macrophage recruitment in the dorsal anterior cingulate white matter of depressed suicides. Brain Behav Immun. 2014:42:50-59.

[45] Morgan JT, et al. Microglial activation and increased microglial density observed in the dorsolateral prefrontal cortex in autism. Biol Psychiatry. 2010:68(4):368-376.

[46] Morgan JT, et al. Stereological study of amygdala glial populations in adolescents and adults with autism spectrum disorder. PLoS One. 2014:9(10):e110356.

[47] Vargas DL, et al. Neuroglial activation and neuroinflammation in the brain of patients with autism. Ann Neurol. 2005:57(1):67-81.

[48] Tetreault NA, et al. Microglia in the cerebral cortex in autism. J Autism Dev Disord. 2012:42(12):2569-2584.

[49] Morgan JT, et al. Abnormal microglial-neuronal spatial organization in the dorsolateral prefrontal cortex in autism. Brain Res. 2012:1456:72-81.

[50] Doorduin $\mathrm{J}$, et al. Neuroinflammation in schizophreniarelated psychosis: A PET study. J Nucl Med. 2009:50(11):1801-1807.

[51] van Berckel BN, et al. Microglia activation in recentonset schizophrenia: A quantitative (R)-[11C]PK11195 positron emission tomography study. Biol Psychiatry. 2008:64(9):820-822.

[52] Takano A, et al. Peripheral benzodiazepine receptors in patients with chronic schizophrenia: A PET study with [11C]DAA1106. Int J Neuropsychopharmacol. 2010:13(7):943-950.

[53] Haarman BC, et al. Neuroinflammation in bipolar disorder - A [(11)C]-(R)-PK11195 positron emission tomography study. Brain Behav Immun. 2014:40:219-225.

[54] Suzuki K, et al. Microglial activation in young adults with autism spectrum disorder. JAMA Psychiatry. 2013:70(1):49-58.

[55] Jones KA, Thomsen C. The role of the innate immune system in psychiatric disorders. Mol Cell Neurosci. 2013:53:52-62.

[56] Liao B, et al. Transformation from a neuroprotective to a neurotoxic microglial phenotype in a mouse model of ALS. Exp Neurol. 2012:237(1):147-152.

[57] Cao L, He C. Polarization of macrophages and microglia in inflammatory demyelination. Neurosci Bull. 2013:29(2):189-198.

[58] Kawai T, Akira S. Signaling to NF-kappaB by Toll-like receptors. Trends Mol Med. 2007:13(11):460-469.

[59] Kobayashi K, et al. Minocycline selectively inhibits M1 polarization of microglia. Cell Death Dis. 2013:4:e525.

[60] Lue LF, et al. Inflammatory repertoire of Alzheimer's disease and nondemented elderly microglia in vitro. Glia. 2001:35(1):72-79.

[61] Ehlermann P, et al. Increased proinflammatory endothelial response to S100A8/A9 after preactivation through advanced glycation end products. Cardiovasc Diabetol. 2006:5:6.
[62] Sunahori K, et al. The S100A8/A9 heterodimer amplifies proinflammatory cytokine production by macrophages via activation of nuclear factor kappa B and p38 mitogenactivated protein kinase in rheumatoid arthritis. Arthritis Res Ther. 2006:8(3):R69.

[63] Nacken W, et al. S100A9/S100A8: Myeloid representatives of the $\mathrm{S} 100$ protein family as prominent players in innate immunity. Microsc Res Tech. 2003:60(6):569-580.

[64] Tubiana R. The clinical manifestations of intrinsic muscle paralysis of the fingers (author's transl). Ann Chir. 1975:29(11):983-985.

[65] Guillemin GJ, et al. Expression of indoleamine 2,3dioxygenase and production of quinolinic acid by human microglia, astrocytes, and neurons. Glia. 2005:49(1):15-23.

[66] Toyooka K, et al. A decrease in interleukin-1 receptor antagonist expression in the prefrontal cortex of schizophrenic patients. Neurosci Res. 2003:46(3):299-307.

[67] Dean B, et al. Different changes in cortical tumor necrosis factor-alpha-related pathways in schizophrenia and mood disorders. Mol Psychiatry. 2013:18(7):767-773.

[68] Kim WK, Ko KH. Potentiation of N-methyl-D-aspartatemediated neurotoxicity by immunostimulated murine microglia. J Neurosci Res. 1998:54(1):17-26.

[69] Busse M, et al. Decreased quinolinic acid in the hippocampus of depressive patients: Evidence for local anti-inflammatory and neuroprotective responses? Eur Arch Psychiatry Clin Neurosci. 2015:265(4):321-329.

[70] Dean B, et al. Regionally-specific changes in levels of tumour necrosis factor in the dorsolateral prefrontal cortex obtained postmortem from subjects with major depressive disorder. J Affect Disord. 2010:120(1-3):245-248.

[71] Ahmed Z, et al. Actin-binding proteins coronin-1a and IBA-1 are effective microglial markers for immunohistochemistry. J Histochem Cytochem. 2007:55(7):687-700.

[72] Wesseling H, Gottschalk MG, Bahn S. Targeted multiplexed selected reaction monitoring analysis evaluates protein expression changes of molecular risk factors for major psychiatric disorders. Int J Neuropsychopharmacol. 2015:18(1).

[73] Pandey GN, et al. Proinflammatory cytokines in the prefrontal cortex of teenage suicide victims. J Psychiatr Res. 2012:46(1):57-63.

[74] Tonelli LH, et al. Elevated cytokine expression in the orbitofrontal cortex of victims of suicide. Acta Psychiatr Scand. 2008:117(3):198-206.

[75] Dwivedi Y, et al. Altered gene expression of brain-derived neurotrophic factor and receptor tyrosine kinase B in postmortem brain of suicide subjects. Arch Gen Psychiatry. 2003:60(8):804-815.

[76] Pandey GN, et al. Brain-derived neurotrophic factor and tyrosine kinase $\mathrm{B}$ receptor signalling in post-mortem brain of teenage suicide victims. Int $\mathbf{J}$ Neuropsychopharmacol. 2008:11(8):1047-1061.

[77] Krishnaswami SR, et al. Using single nuclei for RNA-seq to capture the transcriptome of postmortem neurons. Nat Protoc. 2016:11(3):499-524.

[78] Pietersen CY, et al. Molecular profiles of parvalbuminimmunoreactive neurons in the superior temporal cortex in schizophrenia. J Neurogenet. 2014:28(1-2):70-85.

[79] Kang HJ, et al. Decreased expression of synapse-related genes and loss of synapses in major depressive disorder. Nat Med. 2012:18(9):1413-1417.

[80] Hashimoto T, et al. Gene expression deficits in a subclass of GABA neurons in the prefrontal cortex of subjects with schizophrenia. J Neurosci. 2003:23(15):6315-6326. 
[81] Hemby SE, et al. Gene expression profile for schizophrenia: Discrete neuron transcription patterns in the entorhinal cortex. Arch Gen Psychiatry. 2002:59(7):631-640.

[82] Hakak Y, et al. Genome-wide expression analysis reveals dysregulation of myelination-related genes in chronic schizophrenia. Proc Natl Acad Sci USA. 2001:98(8):47464751.

[83] Segal D, Schmitz C, Hof PR. Spatial distribution and density of oligodendrocytes in the cingulum bundle are unaltered in schizophrenia. Acta Neuropathol. 2009:117(4):385-394.

[84] Yu Z, et al. Therapeutic concentration of lithium stimulates complement $\mathrm{C} 3$ production in dendritic cells and microglia via GSK-3 inhibition. Glia. 2015:63(2):257-270.
[85] Osterndorff-Kahanek EA, et al. Chronic ethanol exposure produces time- and brain region-dependent changes in gene coexpression networks. PLoS One. 2015:10(3): $\mathrm{e} 0121522$.

[86] Lawson LJ, Perry VH, Gordon S. Turnover of resident microglia in the normal adult mouse brain. Neuroscience. 1992:48(2):405-415.

[87] Tomita $\mathrm{H}$, et al. Effect of agonal and postmortem factors on gene expression profile: Quality control in microarray analyses of postmortem human brain. Biol Psychiatry. 2004:55(4):346-352. 